\title{
MORBIMORTALIDADE HOSPITALAR POR EPILEPSIA: ANÁLISE DE DADOS OFICIAIS ${ }^{1}$
}

\author{
Leandro Januário de Lima \\ Raquel Carlos de Brito \\ Maria do Carmo Andrade Duarte de Farias ${ }^{2}$
}

\begin{abstract}
RESUMO. O objetivo deste trabalho foi descrever o perfil clínico-epidemiológico da morbimortalidade hospitalar da Epilepsia nas macrorregiões brasileiras. Trata-se de um estudo descritivo, de cunho quantitativo, epidemiológico retrospectivo, de série temporal, do tipo Ecológico. O objeto de estudo foram os dados sobre morbimortalidade hospitalar entre os anos de 2010 e 2014, disponíveis nos bancos de dados do Ministério da Saúde. Os dados apresentaram uma pequena redução nos internamentos, que passaram de 47819, no ano de 2010, para 46493 ao final da série, em 2014. A taxa de mortalidade hospitalar brasileira variou de 2,26 para 1,97 no período estudado. Em cinco anos, mais de 130 mil hospitalizações no país ocorreram em leitos do setor público, com quase 110 mil no setor privado. O pico dos casos encontra-se nos pequenos infantes, entre um e quatro anos de idade. O padrão de redução dos casos na adolescência, com nova elevação da prevalência entre a terceira e quinta década de vida se repete nas cinco macrorregiões do país. Conclui-se que o estudo das características clínico-epidemiológicas da morbimortalidade hospitalar da Epilepsia nas macrorregiões brasileiras auxilia na compreensão das desigualdades regionais e na proposição de novas estratégias de fomento do sistema único de saúde.
\end{abstract}

Palavras-chave: Perfil de Saúde; Hospitalização; Epilepsia.

\section{HOSPITAL MORBIMORTALITY FOR EPILEPSY: ANALYSIS OF OFFICIAL DATA}

\begin{abstract}
The objective of this study was to describe the clinical-epidemiological profile of hospital morbimortality of epilepsy in the Brazilian macro-regions. This is a descriptive, quantitative, epidemiological retrospective, time series, Ecological type study. The study object was the data on hospital morbimortality between the years 2010 and 2014, available in the databases of the Ministry of Health. The data presented a small reduction in hospitalizations, from 47819 in 2010 to 46493 end of the series in 2014. The Brazilian hospital mortality rate ranged from 2.26 to 1.97 in the period studied. In five years, more than 130,000 hospitalizations in the country occurred in public-sector beds, with nearly 110,000 in the private sector. The peak of cases is found

\footnotetext{
1 O presente trabalho não contou com apoio financeiro de nenhuma natureza para sua realização.

${ }^{2}$ Universidade Federal de Campina Grande (UFCG)
} 
in infants between one and four years of age. The pattern of reduction of cases in adolescence, with a new increase in prevalence between the third and fifth decade of life is repeated in the five macro regions of the country. It is concluded that the study of the clinical-epidemiological characteristics of hospital morbimortality of epilepsy in the Brazilian macro-regions helps to understand regional inequalities and to propose new strategies to promote the single health system.

Keywords: Health Profile; Hospitalization; Epilepsy.

\section{MORBIMORTALIDAD HOSPITALARIA POR EPILEPSIA: ANÁLISIS DE DATOS OFICIALES}

RESUMEN. El objetivo de este trabajo fue describir el perfil clínico-epidemiológico de la morbimortalidad hospitalaria de la Epilepsia en las macrorregiones brasileñas. Se trata de un estudio descriptivo, de cuño cuantitativo, epidemiológico retrospectivo, de serie temporal, del tipo Ecológico. El objetivo de estudio fueron los datos sobre morbimortalidad hospitalaria entre los años 2010 y 2014, disponibles en los bancos de datos del Ministerio de Salud. Los datos presentaron una pequeña reducción en las internaciones, que pasaron de 47819, en el año 2010, a 46493 al año final de la serie, en 2014. La tasa de mortalidad hospitalaria brasileña varía de 2,26 a 1,97 en el período estudiado. En cinco años, más de 130 mil hospitalizaciones en el país se produjeron en lechos del sector público, con casi 110.000 en el sector privado. El pico de los casos se encuentra en los pequeños infantes, entre uno y cuatro años de edad. El patrón de reducción de los casos en la adolescencia, con nueva elevación de la prevalencia entre la tercera y quinta década de vida se repite en las cinco macrorregiones del país. Se concluye que el estudio de las características clínico-epidemiológicas de la morbimortalidad hospitalaria de la Epilepsia en las macrorregiones brasileñas auxilia en la comprensión de las desigualdades regionales y en la proposición de nuevas estrategias de fomento del sistema único de salud.

Palabras claves: Perfil de Salud; Hospitalización; Epilepsia.

\section{Introdução}

Epidemiologicamente, a Epilepsia é definida como a manutenção de um estado de crises epilépticas recorrentes, com as causas orgânicas descartadas. É um distúrbio eletrofisiológico complexo, com manifestações clínicas variáveis, que são enquadradas dentro de um complexo sindrômico (BERTOLUCCI et al., 2011).

É uma condição prevalente na população mundial, afetando todas as faixas etárias. Em estimativas da organização mundial de saúde, entre 4 e 10 pessoas, em cada mil, possuem epilepsia, cerca de 50 milhões em todo o globo. A condição também é 
envolta de estigma social e impactos socioeconômicos (SELLA; SEISDEDOS; BARROS, 2016). Estudos apontam que a epilepsia grave é a quarta condição global nas cargas de morbidades, com uma prevalência na infância maior que o dobro na população adulta em geral (ZUBERY; SYMONDS, 2015).

As análises epidemiológicas desta morbidade ainda são incipientes no Brasil e no Mundo. Como as delimitações são diversas e com variações nos critérios de inclusão e exclusão dos participantes, a comparação dos dados e índices propostos fica prejudicada. Por este cenário, os determinantes sociais da Epilepsia ainda são obscuros (NUNES; GEIB, 2011).

Neste sentido, ao utilizar-se dos dados do Ministério da Saúde é possível traçar um perfil de saúde destes pacientes. Assim, decidiu-se responder neste estudo: qual o perfil de morbimortalidade hospitalar da epilepsia nas macrorregiões brasileiras?

\section{Objetivos}

\section{Objetivo Geral}

Descrever o perfil clínico-epidemiológico da morbimortalidade hospitalar da Epilepsia nas macrorregiões brasileiras.

\section{Objetivos Específicos}

- Caracterizar o regime de atendimento mais prevalente.

- Identificar as faixas etárias com o maior número de casos.

- Identificar as características sociodemográficas dos casos de hospitalização por Epilepsia.

\section{Método}

Trata-se de uma pesquisa descritiva, de cunho quantitativo, classificada como epidemiológica retrospectiva de série temporal, do tipo Ecológica (HULLEY et al., 
2015). O objeto de estudo foram os dados sobre hospitalização e mortalidade hospitalar entre os anos de 2010 e 2014, disponíveis nos bancos de dados do Ministério da Saúde.

A população-alvo componente foi a residente nas macrorregiões brasileiras, que respondem por quase a totalidade do território nacional, que é completado pelos territórios ultramarinos. Segundo o último censo do Instituto Brasileiro de Geografia e Estatística (IBGE) realizado em 2010, a população das macrorregiões correspondia na sua totalidade em 190.755.799 habitantes, encontrando 15.864.454, 53.081.950, 80.364.410, 27.386.891, 14.058.094, para as regiões Norte, Nordeste, Sudeste, Sul e Centro-Oeste (IBGE, 2010).

Os dados de morbimortalidade hospitalar foram obtidos do Sistema de Informações Hospitalares (SIH) do Departamento de Informática do SUS (DATASUS), sistema que é alimentado pelo preenchimento da Autorização de Internação Hospitalar (AIH). Para o cálculo das taxas de prevalência se utilizaram os dados censitários do IBGE e suas projeções oficiais. Em virtude da utilização de dados secundários, esta pesquisa dispensou a submissão ao Comitê de Ética em Pesquisa (BRASIL, 2012; BRASIL, 2016). Os dados foram analisados pela estatística descritiva simples, demonstrados em gráficos e tabela, discutidos à luz da literatura.

\section{Resultados}

Ao longo da série temporal estudada, observa-se uma tendência de estabilidade no número de hospitalizações, apresentada por uma pequena redução nos internamentos que passaram de 47819, no ano de 2010, para 46493, ao final da série, em 2014 (Gráfico 1). Esta estabilidade é vista graças a peculiaridade de que as macrorregiões não apresentarem um padrão único de tendência, pois, enquanto Sudeste, Sul e Centro-oeste evoluíram com redução nas internações por epilepsia, as demais cursaram com aumento.

RPI Revista de Pesquisa Interdisciplinar, Cajazeiras, v. 3, n. 1, 120-130, jan/jul. de 2018. 


\section{Gráfico 1 - Internamentos por Epilepsia nas Macrorregiões - Brasil 2010-2014.}

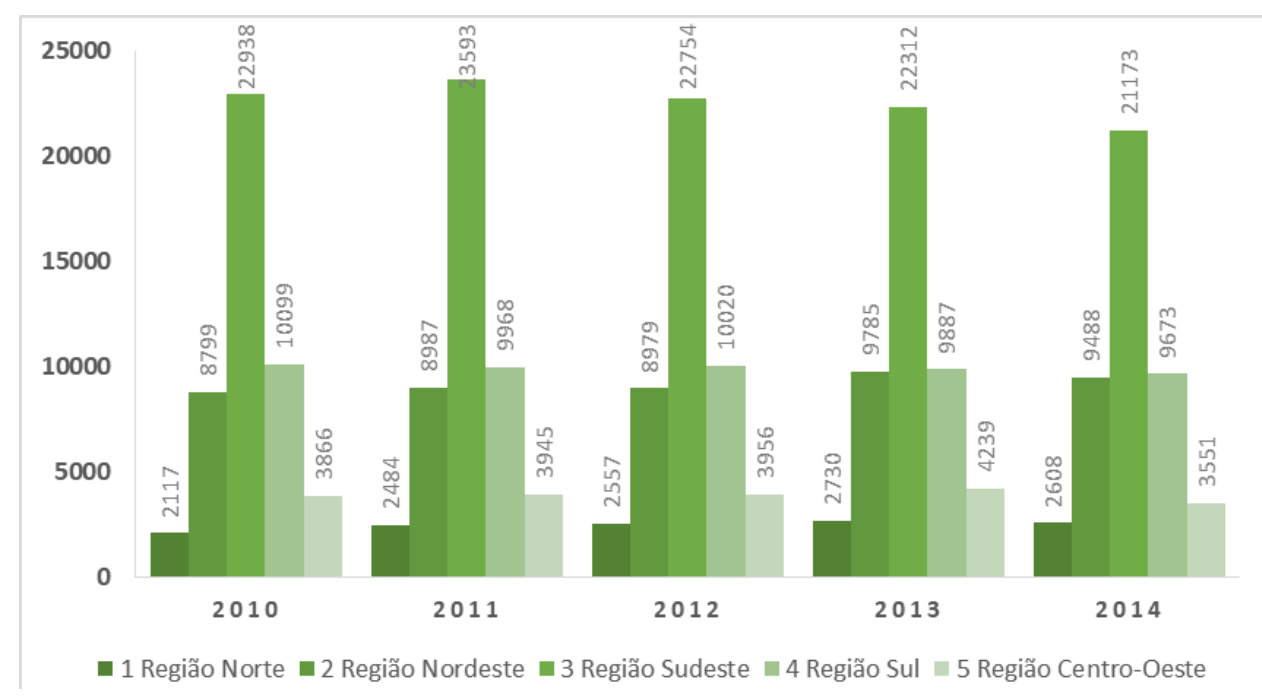

Fonte: Ministério da Saúde - Sistema de Informações Hospitalares do SUS (SIH/SUS).

A taxa de mortalidade hospitalar brasileira da epilepsia, variou de 2,26 para 1,97 no período estudado. A região nordestina apresentou sempre taxa superior a nacional (Gráfico 2). Em 2012, quando o Nordeste apresentou a maior taxa da série com 3,73, seu índice foi $73,5 \%$, maior que o nacional do mesmo período. Apenas a região sudeste também apresentou números de mortalidade superior ao número nacional. Observa-se uma tendência na redução dos óbitos por epilepsia na média nacional, embora os dados nas macrorregiões não se comportem de maneira equânime. A mortalidade dos pacientes por epilepsia foi maior que a população em geral, sem uma relação direta com o pós-operatório da cirurgia para correção com esta morbidade (GOMES, 2010). A redução da taxa de mortalidade no período está em continuidade com o observado entre 1980 e 2003, condizente com o padrão internacional (FERREIRA; SILVA, 2009). 


\section{Gráfico 2 - Taxa de Mortalidade Hospitalar por Epilepsia - Brasil 2010-2014.}

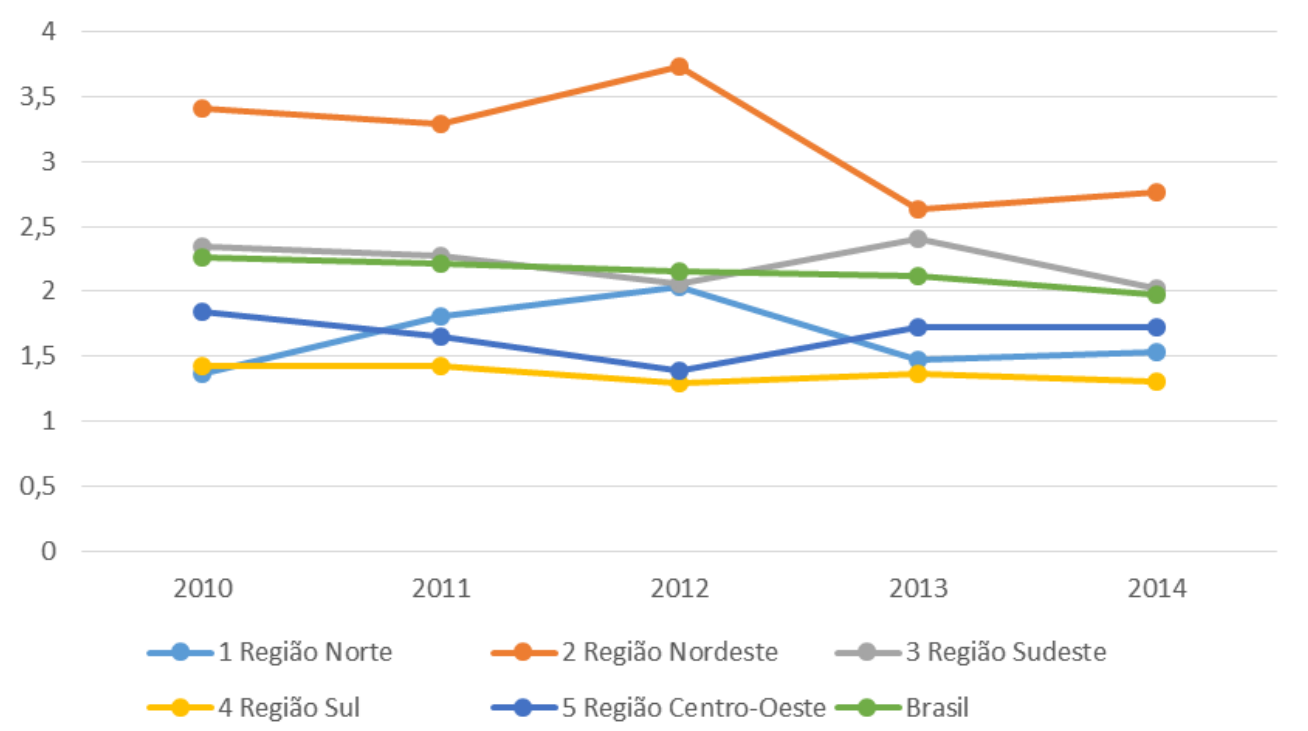

Fonte: Ministério da Saúde - Sistema de Informações Hospitalares do SUS (SIH/SUS).

Em 2010, foram investidos $\mathrm{R} \$ 21.433 .817,68$ nos internamentos com a causa principal Epilepsia (Tabela 1). O aumento na série foi de 7,97\%, com um investimento de $\mathrm{R} \$ 23.143 .583,81$ no quantitativo nacional, com o mesmo padrão de redução dos internamentos sendo observado nos investimentos, sendo esses o seu principal determinante. O Nordeste, que apresentou a maior taxa de mortalidade, teve em toda a série o segundo menor valor médio de internamento. Sudeste, Sul e Centro-Oeste, que se mantiveram na ponta da tabela com os maiores valores por hospitalização, observaram uma queda nos valores até 2012, retomando uma elevação dos custos, a partir de 2013 (Gráfico 3).

Tabela 1 - Investimentos nas Hospitalizações por Epilepsia nas Macrorregiões -

Brasil 2010-2014.

\begin{tabular}{lrrrrr}
\hline Região / Ano & $\mathbf{2 0 1 0}$ & $\mathbf{2 0 1 1}$ & $\mathbf{2 0 1 2}$ & $\mathbf{2 0 1 3}$ & $\mathbf{2 0 1 4}$ \\
\hline Norte & $562.729,24$ & $730.751,04$ & $743.088,34$ & $848.283,74$ & $832.106,06$ \\
Nordeste & $2.701 .930,44$ & $3.116 .953,53$ & $3.489 .036,23$ & $3.984 .002,16$ & $4.312 .913,81$ \\
Sudeste & $12.579 .215,36$ & $12.007 .690,02$ & $11.272 .363,88$ & $12.542 .487,16$ & $11.464 .832,73$ \\
Sul & $4.148 .982,65$ & $4.258 .493,59$ & $4.418 .692,10$ & 4976285,61 & $4.941 .064,15$ \\
Centro-Oeste & $1.440 .959,99$ & $1.397 .942,46$ & $1.605 .917,24$ & $1.701 .272,23$ & $1.592 .667,06$
\end{tabular}

Fonte: Ministério da Saúde - Sistema de Informações Hospitalares do SUS (SIH/SUS).

RPI Revista de Pesquisa Interdisciplinar, Cajazeiras, v. 3, n. 1, 120-130, jan/jul. de 2018. 


\section{Gráfico 3 - Investimento Médio nas Hospitalizações por Epilepsia - Brasil 2010-} 2014.

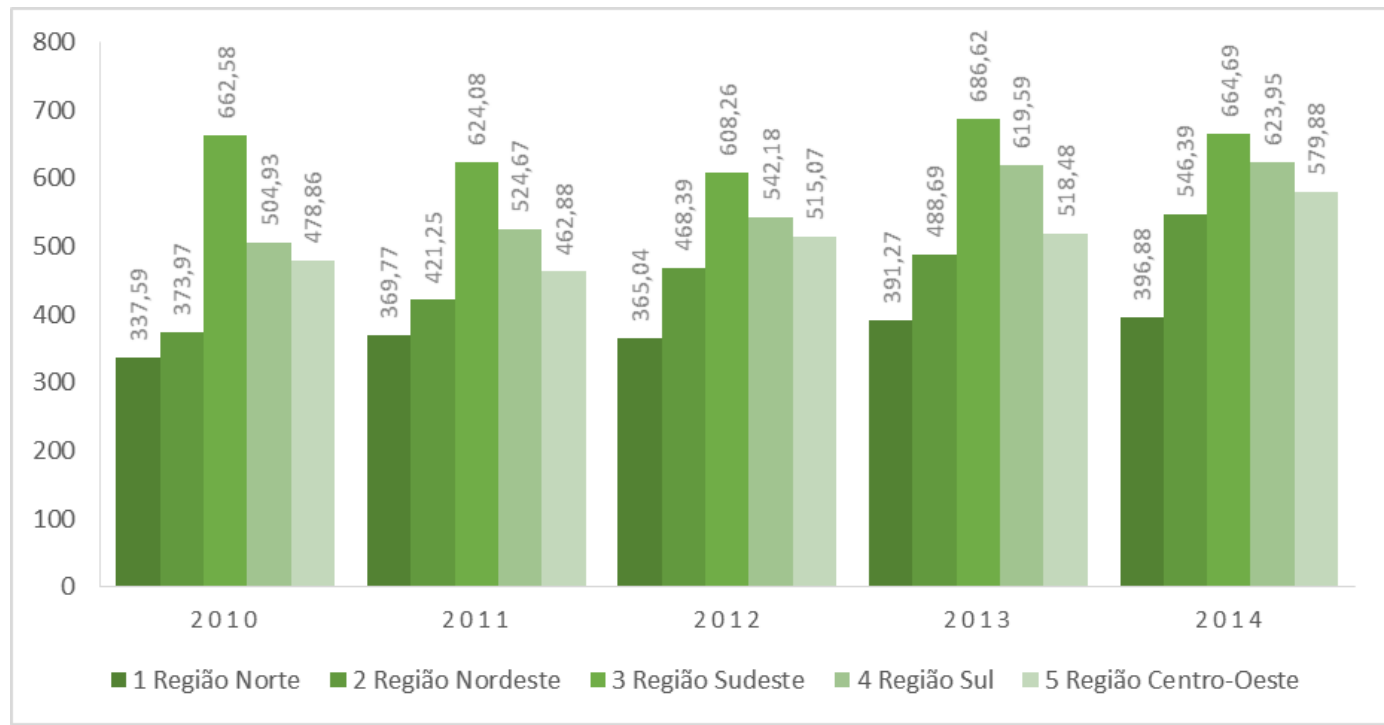

Fonte: Ministério da Saúde - Sistema de Informações Hospitalares do SUS (SIH/SUS).

Em cinco anos, mais de 130 mil hospitalizações no país ocorreram em leitos do setor público, com quase 110 mil no setor privado (Gráfico 4). De todas as macrorregiões, apenas a Sul teve um perfil assistencial no período concentrado no setor privado. As autorizações de internação hospitalar, cujo dado do regime no sistema foi ignorado, equivale a $0,002 \%$ do quantitativo do período. Salienta-se as disparidades regionais com a constatação de que no Norte do país a dependência dos leitos públicos foi de $91,7 \%$, nas hospitalizações por Epilepsia. 


\section{Gráfico 4 - Internamentos por Regime de Atendimento - Brasil 2010-2014.}

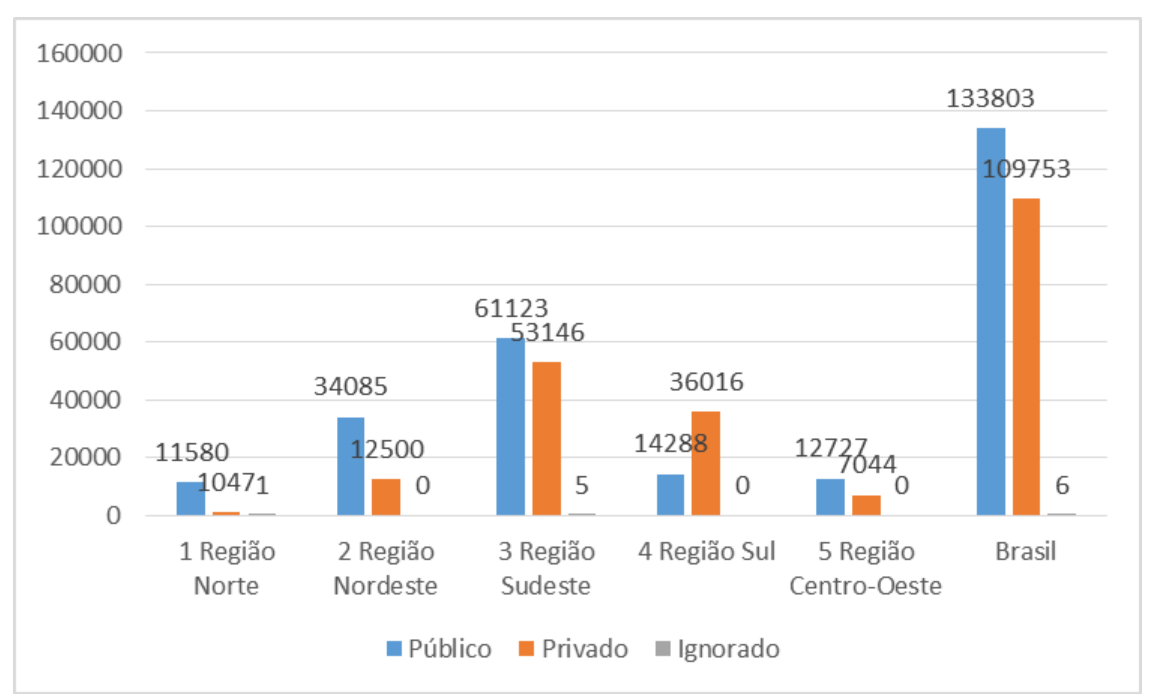

Fonte: Ministério da Saúde - Sistemas de Informações Hospitalares do SUS (SIH/SUS).

A urgência foi o caráter de atendimento primordial da hospitalização por epilepsia. O perfil nacional do período foi de que $92,3 \%$ dos internamentos nesta modalidade. Apenas o Nordeste, com 96,2\%, e o Centro-oeste que se apresentou com 95,9\% das hospitalizações neste caráter superaram a porcentagem nacional (Gráfico 5). Embora seja a região que mais dependeu do setor privado, nos internamentos por epilepsia, a região Sul foi a segunda com menos internamentos eletivos, com taxa menor apenas para o Norte, com 1289 casos.

Os dados tornam difícil uma relação casuística, mesmo que preliminar, entre a epilepsia e um padrão de cor dos indivíduos. Neste quesito, aparece a fragilidade dos dados secundários, pois, nas regiões Nordeste e Centro-oeste o número de internamentos cuja cor do paciente não foi colhida na AIH ou repassada aos bancos de dados representam o maior quantitativo dentro da série. Na região Norte, foi o segundo maior, situação presente em 5199 internamentos. No Sul e Sudeste, a cor branca foi a principal nos quadros de hospitalização por epilepsia, com a cor parda a $3^{\mathrm{a}}$ mais prevalente em ambas (Gráfico 6). 


\section{Gráfico 5 - Internamentos por Caráter de Atendimento - Brasil 2010-2014.}

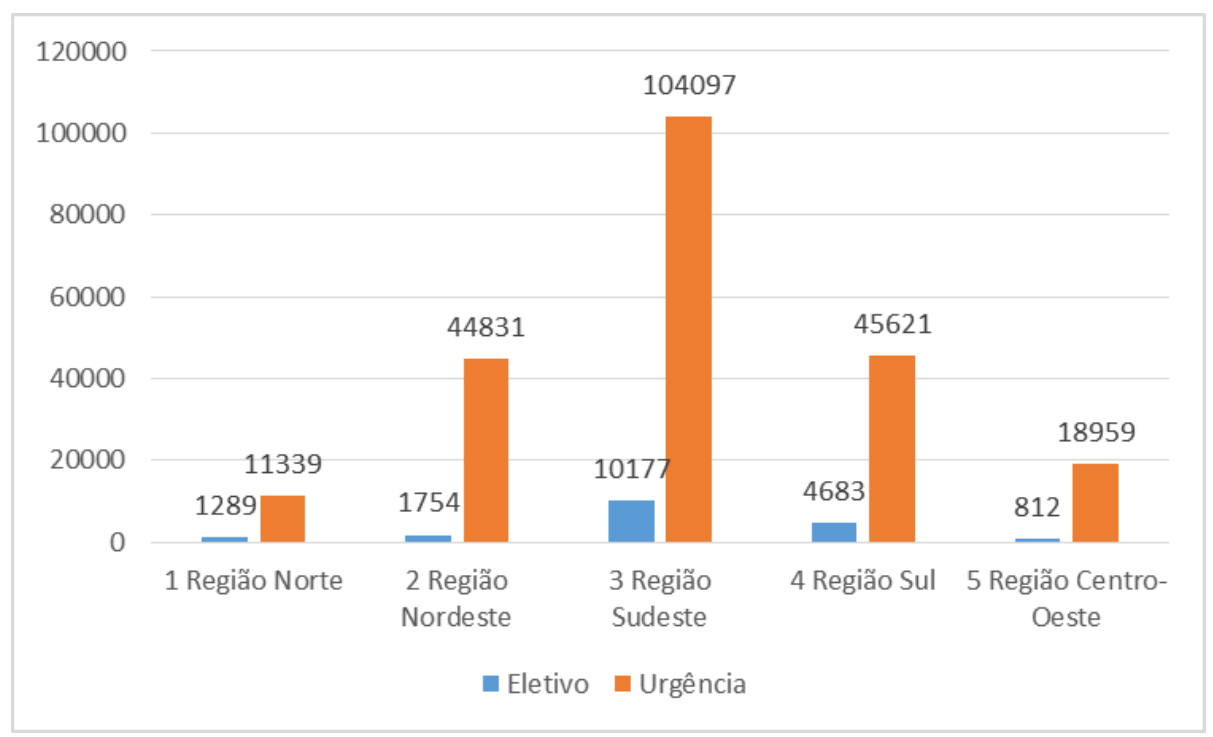

Fonte: Ministério da Saúde - Sistema de Informações Hospitalares do SUS (SIH/SUS).

Gráfico 6 - Internamentos por Cor - Brasil 2010-2014.

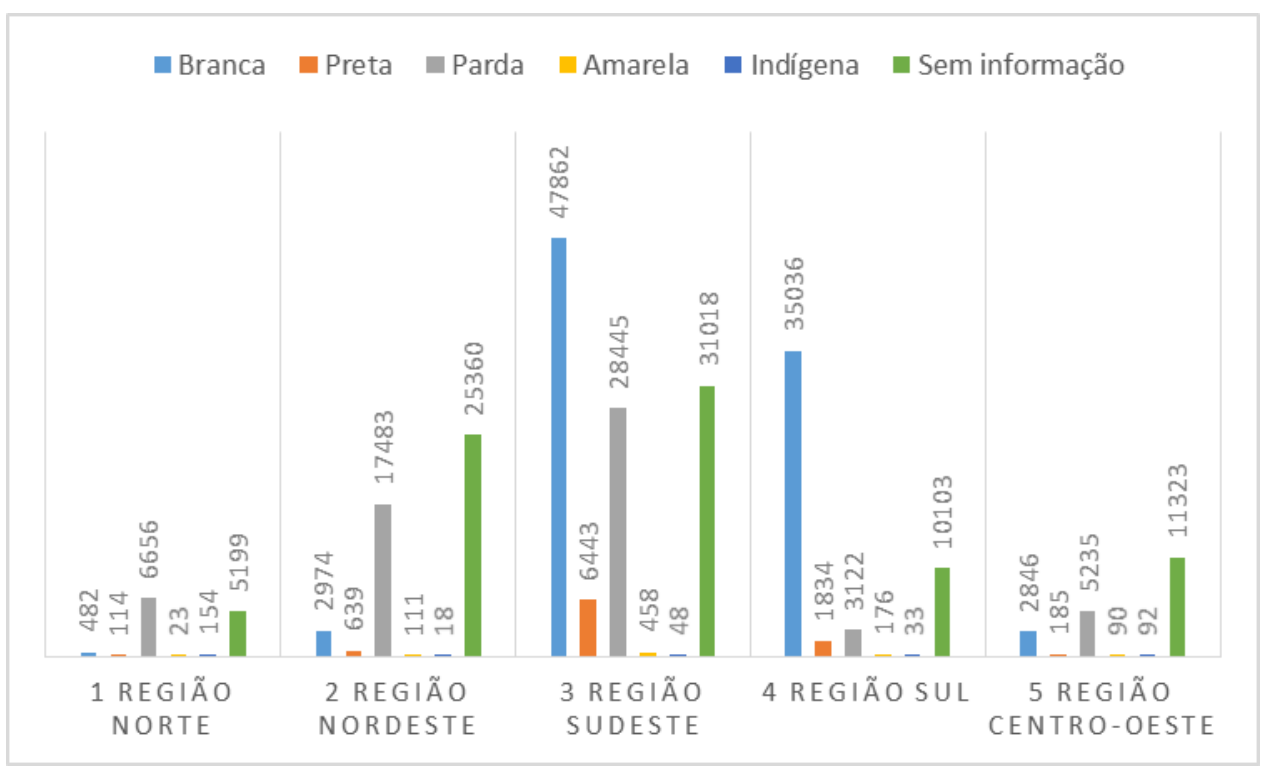

Fonte: Ministério da Saúde - Sistema de Informações Hospitalares dos SUS (SIH/SUS).

O pico dos casos encontrou-se nos pequenos infantes entre um e quatro anos de idade. O padrão de redução dos casos na adolescência, com nova elevação da prevalência entre a terceira e quinta década de vida, se repete nas cinco macrorregiões (Gráfico 7). O menor número de casos se encontrou nos pacientes idosos com 80 anos de idade ou mais. Nos países desenvolvidos, vem se observado um aumento nos número 
de casos de epilepsia nos idosos com mais de 60 anos (GOMES, 2012). A incidência de epilepsia em crianças encontrada em um hospital brasileiro superou as taxas de países desenvolvidos (ALVES-LEON et al., 2009). Em menores de um ano, a principal epilepsia encontrada foram os espasmos infantis (ZUKERMAN; BRANDT, 2011).

\section{Gráfico 7 - Internamentos por faixa etária - Brasil 2010-2014.}

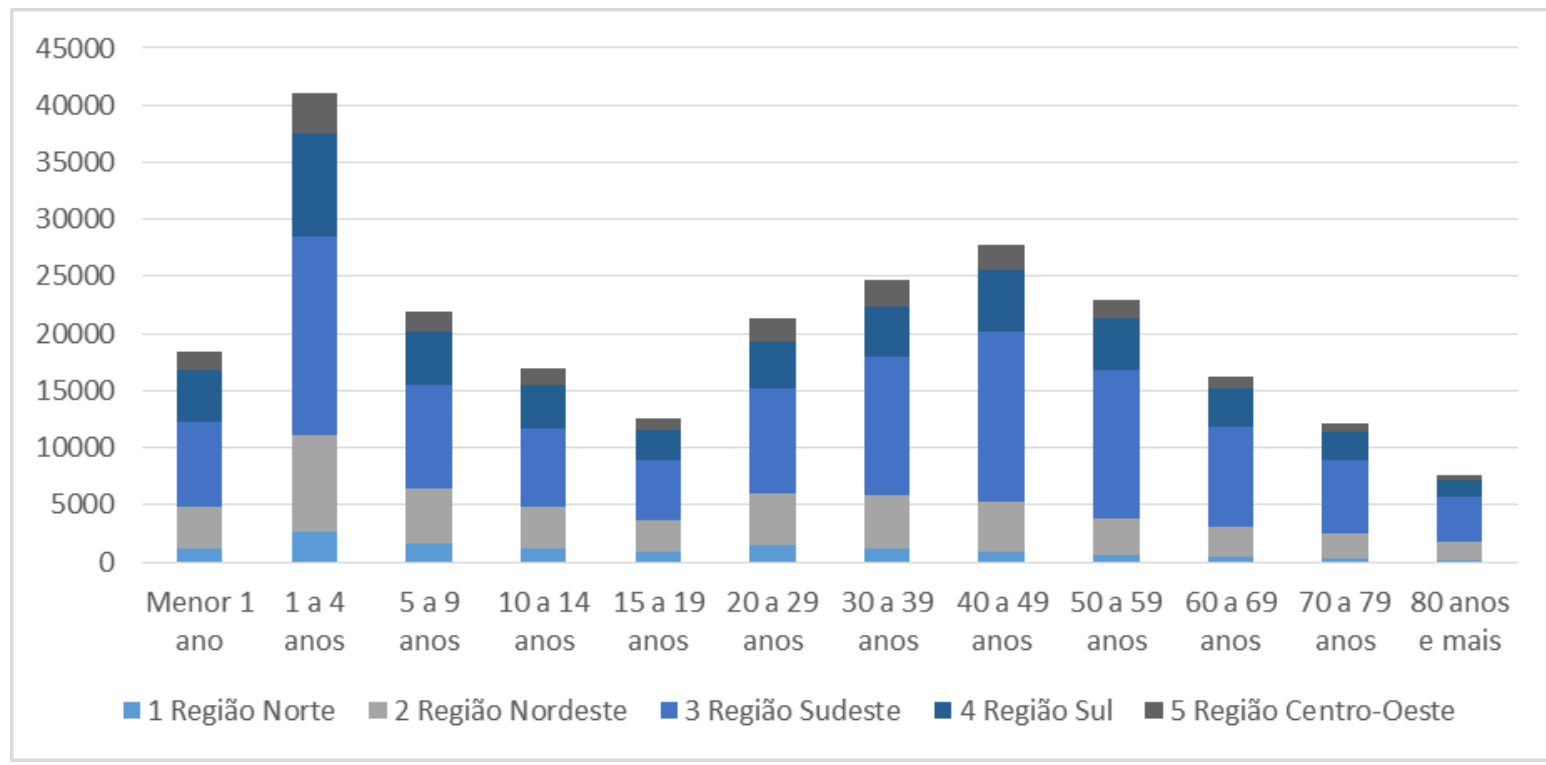

Fonte: Ministério da Saúde - Sistema de Informações Hospitalares do SUS (SIH/SUS).

No período, os internamentos por Epilepsia em todas as macrorregiões foram mais comuns no sexo masculino. O perfil nacional foi de 58,46\% dos casos com pacientes do sexo masculino. Na região Sul, observou-se o maior percentual com 59,9\% dos pacientes, sendo a única macrorregião nacional a superar o padrão nacional (Gráfico 8). Em contraste, no Norte do país encontrou-se a menor taxa com 56,21\% dos usuários declarando o sexo masculino. 


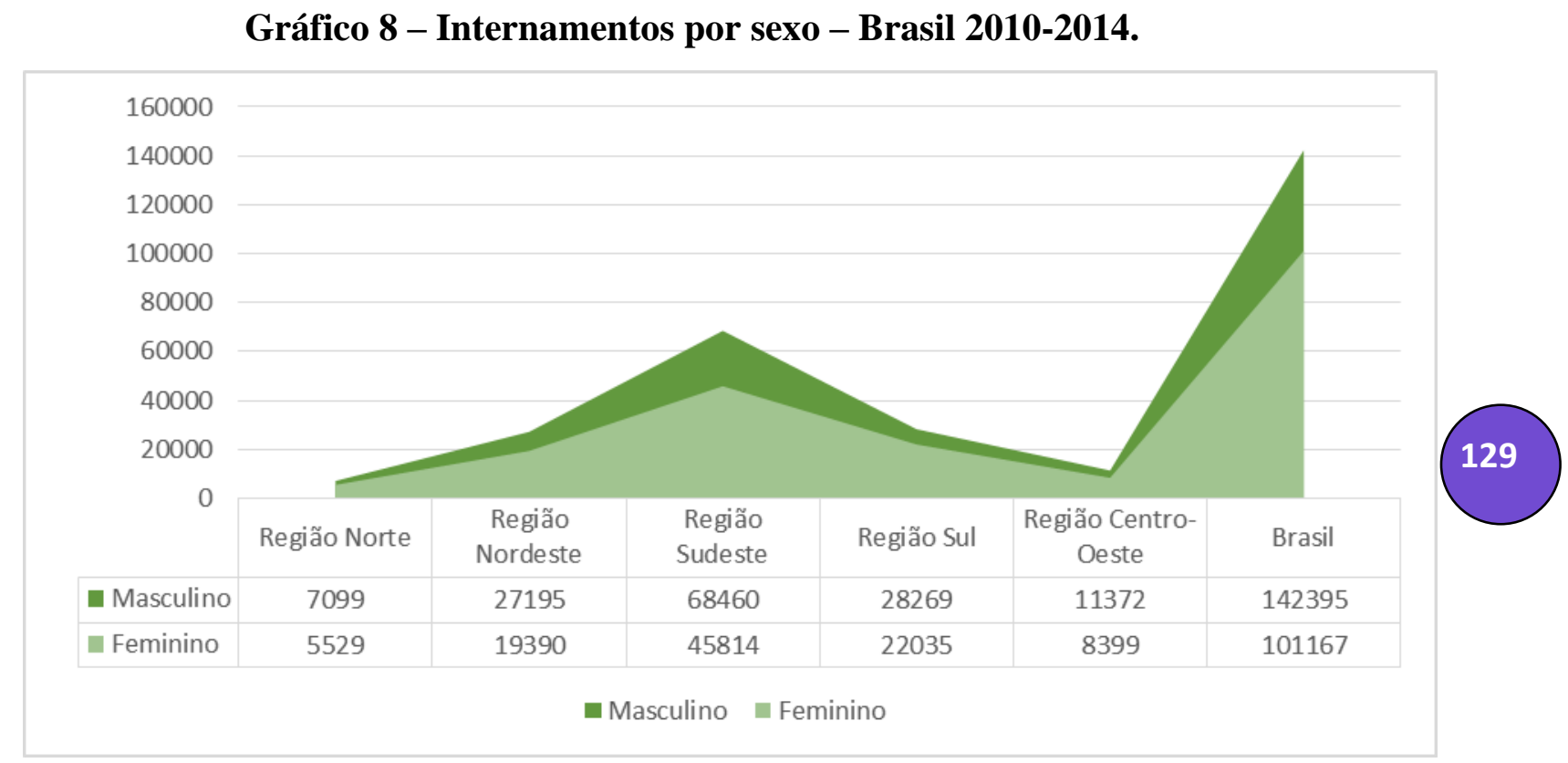

Fonte: Ministério da Saúde - Sistema de Informações Hospitalares do SUS (SIH/SUS).

\section{Conclusão}

Encontrou-se um perfil de pacientes hospitalizados que são em sua maioria menores de 10 anos, do sexo masculino, que chegam as instituições de saúde com caráter de urgência, principalmente em hospitais públicos. A caracterização deste perfil assistencial favorece o planejamento de ações que possam proporcionar o diagnóstico precoce e o tratamento correto dessa condição tão prevalente em nossa população.

O estudo das características clínico-epidemiológicas da morbimortalidade hospitalar da Epilepsia nas macrorregiões brasileiras auxilia na compreensão das desigualdades regionais e na proposição de novas estratégias de fomento dos sistema único de saúde.

\section{Conflito de interesses}

Em atendimento às resoluções do Conselho Federal de Medicina n ${ }^{\circ}$ 1.595/2000 e $n^{\text {o }}$ 1.974/2011 e à resolução RDC nº 96 de 2008 da Agência Nacional de Vigilância Sanitária, os autores declaram não haver nenhum conflito de interesse com a temática abordada. 


\section{Referências}

ALVES-LEON, S. V. et al. Crises epilépticas no período neonatal: análise descritiva de uma população hospitalar. Rev. paul. pediatr., São Paulo, v. 27, n. 2, p. 173-178, Jun. 2009.

BERTOLUCCI, P. F. H. et al. (Ed.). Neurologia. São Paulo: Manole, 2011. 1140 p.

BRASIL. Resolução nº 466, de 12 de dezembro de 2012. Diário Oficial da União. Brasília, DF, 2012.

BRASIL. Resolução n ${ }^{\circ}$ 510, de 07 de abril de 2016. Diário Oficial da União. Brasília, DF, 2016.

FERREIRA, I. L. M.; SILVA, T. P. T. Mortalidade por Epilepsia no Brasil, 1980-2003. Ciência \& Saúde Coletiva, [s.1], v. 14, n. 1, p. 89-94, 2009.

GOMES, M. M. Mortalidade atribuída à epilepsia, às suas doenças subjacentes ou às condições não relacionadas a ela: uma síntese. J. epilepsy clin. neurophysiol., Porto Alegre, v. 16, n. 3, p. 100-105, set. 2010.

GOMES, M. M. Prognóstico a Longo Prazo da Epilepsia. J. epilepsy clin. neurophysiol., Porto Alegre, v. 18, n. 2, p. 35-40, 2012.

HULLEY, S. B. et al. Delineando a pesquisa clínica. 4. ed. Porto Alegre: Artmed, 2015. 400 p.

IBGE. Sinopse do Censo Demográfico 2010 - Brasil. Disponível em:

https://censo2010.ibge.gov.br/sinopse/index.php. Acesso em: 28 abr. 2018.

NUNES, M. L.; GEIB, L. T. C. Incidência de epilepsia e distúrbios convulsivos na infância e sua associação com determinantes sociais: um estudo de coorte de nascimento. J. Pediatr. (Rio J.), Porto Alegre, v. 87, n. 1, p. 50-56, fev. 2011.

SELLA, F.; SEISDEDOS, R. A.; BARROS, C. R. M. R. Perfil Epidemiológico dos Membros de uma Associação de Epilepsia em Santa Catarina. Arquivos Catarinenses de Medicina, [S.1.], v. 45, n. 2, p. 59-66, set. 2016.

ZUBERI, S. M.; SYMONDS, J. D. Atualização sobre o diagnóstico e tratamento de epilepsias da infância. J. Pediatr. (Rio J.), Porto Alegre, v. 91, n. 6, supl. 1, p. S67-S77, Dec. 2015.

ZUKERMAN, E.; BRANDT, R. A. Neurologia e Neurocirurgia: a prática clínica e cirúrgica por meio de casos. Barueri, SP: Manole, 2011. 478 p. 